\title{
Initial radiation DNA damage observed in prematurely condensed chromosomes of G2-phase human lymphocytes and analytical model of ion tracks ${ }^{\star}$
}

\author{
Agata Kowalska ${ }^{1, a}$, Konrad Czerski ${ }^{2}$, Elena Nasonova ${ }^{3}$, Polina Kutsalo ${ }^{3}$, and Eugene Krasavin ${ }^{3}$ \\ 1 Department of Physics and Chemistry, Maritime University of Szczecin, Wały Chrobrego 1-2, 70-500 Szczecin, Poland \\ 2 Institute of Physics, University of Szczecin, ul. Wielkopolska 15, 70-451 Szczecin, Poland \\ 3 Joint Institute for Nuclear Research, Joliot-Curie 6, 141980 Dubna, Russia
}

Received 28 February 2019 / Received in final form 20 November 2019

Published online 28 January 2020

(C) The Author(s) 2020. This article is published with open access at Springerlink.com

\begin{abstract}
In the present work, we have adopted the premature chromosome condensation (PCC) technique to study the initial DNA damage in order to distinguish between the biological and physical components of the dose-effect curves. We have studied $\mathrm{G}_{2}$-chromatid breaks in human lymphocytes, isochromatid-type breaks as well as chromatid exchanges at two different sampling times: directly after irradiation $\left(t_{0}\right)$ and 12 hours later $\left(t_{12}\right)$. The lymphocyte samples have been exposed to $150 \mathrm{MeV}$ and spread out Bragg peak (SOBP) proton beams, $22 \mathrm{MeV} / \mathrm{u}{ }^{11} \mathrm{~B}$ ions and for comparison to ${ }^{60} \mathrm{Co} \gamma$ rays. Dose-response curves for both types of breaks have been determined. We have shown that $t_{0} G_{2}$-chromatid breaks follow linearquadratic dependence for all studied cases and could be used for estimation of the effective ion track radius. A comparison to the expected physical track radii leads to the conclusion that the biological repair mechanism considerably prevails the physical effect of the overlapping ion tracks even at the time $t_{0}$. The results have been also compared to the dose-effect curves previously obtained in our chromosome aberrations study.
\end{abstract}

\section{Introduction}

Knowledge of the biological response of single cells to different radiation quality is of fundamental importance not only for protection against radiation, but also for the use of heavy charged particles in radiation therapy [1].

Differences in the response of investigated cells or tissues exposed to ionizing radiation depend on physical properties of chosen particle flux, absorbed dose and dose rate resulting from formation of ion track structures. Scenarios of cell response can be studied in several ways. One of the most frequently used is the survival assay describing the fraction of cells which sustained given radiation doses. More powerful methods, however, rely on the observation of chromosome aberrations (CA) induced in peripheral blood lymphocytes (PBL) [2] which are preferable for several reasons. First of all, the assessment of chromosome damage in human lymphocytes is considered to be the only reliable method in human biodosimetry since half a century [3]. Secondly, lymphocytes circulate in the blood vessels and are distributed throughout the body, and the

* Contribution to the Topical Issue "Dynamics of Systems on the Nanoscale (2018)", edited by Ilko Bald, Ilia A. Solov'yov, Nigel J. Mason and Andrey V. Solov'yov.

a e-mail: a.kowalska@am.szczecin.pl damage to the hematopoietic tissue is a major limiting factor for a total dose in radiotherapy.

So-called dose-response curves of CA, associated to DNA double strand breaks, usually have a linear-quadratic shape [4]. As already shown, the quadratic term may result from two different effects. The most important effect is of a biological origin and can be connected with very efficient DNA repair mechanisms leading to $\mathrm{CA}$ which strongly depend on the local ionization density and thus on the radiation quality $[5,6]$. Additionally, quadratic coefficient may be associated with physical processes of ion track overlapping $[7,8]$ and doubling of the locally absorbed dose. Although, physics concerning interaction of ionizing radiation [9] and the processes of the DNA damage leading to chromosomal aberrations $[5,10]$ are relatively well known, the allencompassing anticipation of the radiobiological outcomes is still missing. The physical models based on Monte Carlo simulations can correctly describe evolution of ion tracks formed in the irradiated tissue by starting with ionization and creation of fast delta electrons which transfer their energy in the next step to the atomic system and finally lead to production of chemically active radicals. These models are, however, unable to assess the final biological effect of irradiation due to the lack of well-defined repair mechanisms, which are very effective [6] and indispensable for description of the $\mathrm{CA}$ formation. 
Existing phenomenological models, like the local effect model (LEM) [11] and the Katz model [12,13], simply assume that the radiation induced damage of the cellular DNA and the corresponding number of double strand breaks (DSB) only depends on the local ionization density, and therefore, the response function obtained for the gamma irradiation can be applied to model dose-effect curves of charged particles. Relatively good agreement with the experimental data is, however, occupied by fitting several free parameters which take on specific values for the cell line actually studied but also depend on the radiation quality. Again, the reason for that is most likely due to the underestimation of the effects of cellular repair leading to the CA formation.

Recently, we have proposed a simple analytical model describing the observed linear-quadratic dependence of the chromosome aberration yield on the radiation dose induced by heavy charged particles [14]. The method is based on the assumption that the non-linear term of linear-quadratic response function arises solely by the ion track overlapping effect. Using the experimentally assessed parameters, we could obtain an effective track radius which could explain experimentally observed curvature of the dose-effect response. Comparison between the effective track radius and the physical expectations led us to the conclusion that the experimentally determined curvature of the dose-effect curves predominantly results from cellular repair effects.

The simplest way to distinguish between the physical overlapping effect and the biological mechanisms, being the main purpose of the present work, is certainly to observe the initial DNA damage, directly after irradiation. Such an experiment is not easy to conduct since the repair mechanisms possess, apart from a long-term component lasting many hours, also a fast contribution of 5-10 minutes during which a part of the DNA breakages can be rejoined [15]. It means that repair takes place already during the irradiation process.

Initial DNA damage can be visualized by several methods, such as gel electrophoresis [16], $\gamma \mathrm{H} 2 \mathrm{AX}$ foci visualization [17] or premature chromosome condensation (PCC) [16]. In the present work we have adopted very fast and reliable PCC technique as already shown in many previous studies [24-27].

Chromosomes condense during mitosis. However, chromatin condensation may be induced at any phase of the cell cycle by means of several agents, leading to so called "premature chromosome condensation". PCC has proved to be a useful tool for analyzing chromosomes in interphase and visualize genetic damage shortly after the radiation exposure. Chromosome condensation by means of Calyculin A, a specific inhibitor of type 1 and $2 \mathrm{~A}$ protein phosphatases [18], is a very simple method which allows to analyze $\mathrm{G}_{1}$ cells (displaying univalent chromosomes), $\mathrm{G}_{2}$ cells (displaying bivalent chromosomes), and visualizes S-phase cells (displaying pulverized mixture of uni- and bivalent pieces of chromatin). Here, we recorded the number of chromatid breaks induced immediately and 12 hours after the exposure to high and low LET radiation in $\mathrm{G}_{2}$ cells.

Our previous studies involving CA observation were based on conventional metaphase method in resting-state human lymphocytes which were irradiated in $G_{0}$ phase of the cell cycle, stimulated to proliferate, and analyzed after $48 \mathrm{~h}$ of culturing. This is the time when culturing lymphocytes reach their first post-irradiated mitosis in the natural way. Thus, initial formation and rejoining studies of chromatid breaks cannot be performed by metaphase method because rejoining of double strand breaks proceeds much faster $[15,19]$. In contrast, chemically induced PCC technique allows to study kinetics of repair mechanisms in a very short time steps according to experimental requirements of irradiation procedure.

\section{Materials and methods}

\subsection{Blood irradiation and cytogenetic analysis}

All blood samples were obtained from informed, healthy volunteers. Ethical approval was obtained for this study and all participants gave informed consent.

Whole blood was obtained by venipuncture into heparinized vacuum containers. The lymphocytes were isolated by gradient centrifugation and seeded with a density of $0.5 \times 10^{6} \mathrm{cell} / \mathrm{ml}$ in the in RPMI medium supplemented by $20 \%$ fetal calf serum, $2 \mathrm{mM}$ L-glutamine, $100 \mathrm{U} / \mathrm{ml}$ penicillin, $100 \mu \mathrm{g} / \mathrm{ml}$ streptomycin and $1 \%$ phytohaemagglutinin (PHA).

After $48 \mathrm{~h}$ of culture, asynchronously growing cell population was exposed to ${ }^{60} \mathrm{Co} \gamma$ rays and heavy ions (150 MeV and SOBP protons, and $22.1 \mathrm{MeV} / \mathrm{u}^{11} \mathrm{~B}$ ions). Proton and $\gamma$ irradiation was performed at the medical complex of Dezheleplov Laboratory of Nuclear Problems of the Joint Institute for Nuclear Research, Dubna, Russia. Protons were delivered by Phasotron; ${ }^{60} \mathrm{Co} \gamma$ ray irradiation was done on the remote radiation therapy unit ROKUS M. Monoenergetic $22.1 \mathrm{MeV} / \mathrm{u}{ }^{11} \mathrm{~B}$ beam was delivered by the $\mathrm{U} 400 \mathrm{M}$ cyclotron of the Flerov Laboratory of Nuclear Reactions, JINR (Dubna, Russia). Details of the irradiation procedure and beam characteristics are given elsewhere [14,20,21].

Immediately after irradiation one set of all samples was treated with $50 \mathrm{nM}$ of Calyculin A for $50-60 \mathrm{~min}$ at $37^{\circ} \mathrm{C}$, then hypotonised with $75 \mathrm{mM} \mathrm{KCl}$, and fixed with methanol: glacial acetic acid. All samples were irradiated separately, and irradiation time amounted to 1-2 minutes. Only in the case of ${ }^{11} \mathrm{~B}$ ions, all 8 biological samples were placed together in a changeable rotary drum [21]. When the drum rotated, each blood sample - one by one - was placed in front of the ion beam which passed through the central collimator. Doses ranged between 0.05 and $2 \mathrm{~Gy}$, and Calyculin A was added immediately after irradiation of the whole set had been finished. The total boron irradiation time (dose rate $1.24 \mathrm{~Gy} / \mathrm{min}$ ) lasted $\sim 5$ min.

The second set of samples, immediately after irradiation was subjected to further incubation at $37^{0} \mathrm{C}$ and after 12 $\mathrm{h}$ was treated with $50 \mathrm{nM}$ of Calyculin A and fixed in the same manner as first set. So prepared material was dropped onto glass slides, dried, stained with $3 \%$ Giemsa in phosphate-buffered saline ( $\mathrm{pH}$ 6.8) and analyzed under an optical microscope. 
a.

\begin{tabular}{|c|c|c|}
\hline $\begin{array}{c}\text { Chromatid } \\
\text { break }\end{array}$ & $\begin{array}{c}\text { Isochromatid } \\
\text { break }\end{array}$ & $\begin{array}{c}\text { Chromatid } \\
\text { exchange }\end{array}$ \\
\hline & & \\
\hline
\end{tabular}

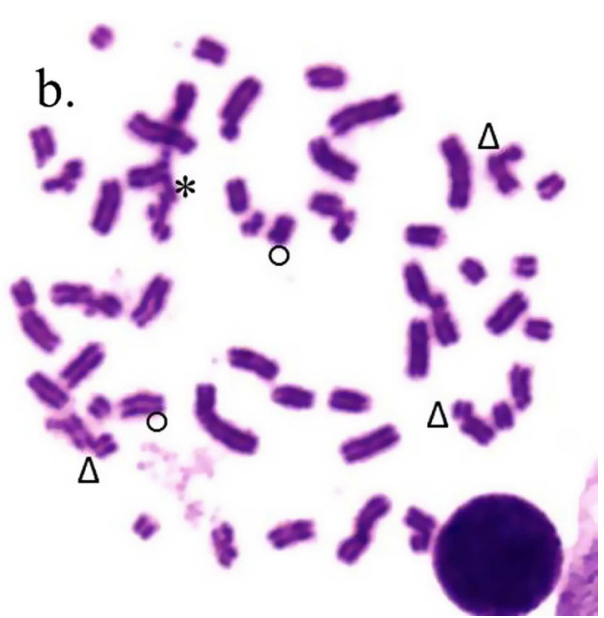

Fig. 1. (a.) Schematic view of the initial damage assessed by the PCC method. Drawings are taken from [28]; (b.) the G2-spread consists of 49 figures, i.e. there are 3 isochromatid breaks, and contains 20 chromatid breaks. Exemplary labeling: isochromatid breaks $(\bigcirc)$, chromatid breaks $(\Delta)$, exchanges $(*)$.

Typically, $100-200 \mathrm{G}_{2}$-phase cells were analyzed for every data point. The damage was classified as chromatid breaks, iso-chromatid breaks (excess figures) and chromatid exchanges (see Fig. 1a) being the sign of prompt DNA repair mechanisms.

The yield of isochromatid breaks was measured from the excess number of chromosomes ( $>46$ figures) observed [3] (see Fig. 1). In G2-phase of the cell cycle the iso-chromatid break occurs when two breaks are formed on the opposite sister chromatids in a close proximity. Because one isochromatid break results from the breakage of both chromatid threats, one isochromatid break was scored as two chromatid breaks.

\subsection{Effective ion track radius}

The yield of breaks $Y$ visible after exposure to different doses of ionizing radiation $D$ can be generally described by the linear-quadratic model:

$$
Y=\alpha \cdot D+\beta \cdot D^{2} .
$$

As discussed in our previous paper [14] devoted to the chromosome aberrations of the human lymphocytes, the quadratic term can represent contributions resulting both from the physical effect of the overlapping ion tracks and from the biological repair mechanisms which effectiveness is not known exactly. Thus, we have proposed to determine the curvature ratio $\beta / \alpha$ theoretically, assuming only that the ion tracks of an effective interaction radius $R_{\text {eff }}$ can overlap and produce locally in this overlapping region a double dose. As the ion tracks are distributed over the irradiated cell statistically and the distance between them follows the two dimensional Poisson statistics, the $\beta / \alpha$ ratio can be related to the effective track radius as follows [14]:

$$
R_{\exp }=\sqrt{\frac{3 \cdot L E T}{8 \cdot F \cdot \rho_{m}}} \cdot \sqrt{\frac{\beta}{\alpha}}
$$

where $F$ is a geometric factor equal to 0.58 describing the overlapping region, $\rho_{m}$ denotes the mass density of the cell, and $L E T$ is the LET value of the irradiation applied. Since the $\beta / \alpha$ ratio is easy to determine from experimental dose-effect curves, we can calculate using the formula above how large should be the interaction radius of the ion tracks to explain the experimentally observed curvature of the response function. Therefore, we can speak about the experimental effective track radius.

On the other hand, we can also determine a physical effective ion track radius using the known structure of ion tracks and the range of fast $\delta$ electrons produced during the ionization process induced by slowing down ions. Assuming a constant radial dose density within the ion track, we get the following expression for the physical effective track radius [14]:

$$
R_{\text {phys }}=R_{\min } \sqrt{1+2 \ln \frac{R_{\max }}{R_{\min }}}
$$

where $R_{\min }$ corresponds to the track core radius and $R_{\max }$ stands for track penumbra radius of the $1 / \mathrm{r}^{2}$ declining radial dose, determined by the range of the $\delta$ electrons. The quantities above can be calculated according to the formula proposed by Chatterjee and Schaefer, 1976 [22]:

$$
\begin{aligned}
R_{\min } & =\frac{v}{c} \cdot 0.0116(\mu \mathrm{m}) \\
R_{\max } & =0.768 E-1.925 \sqrt{E}+1.257 \mu \mathrm{m} .
\end{aligned}
$$

Here $E$ stands for particle's kinetic energy per nucleon in $\mathrm{MeV} / \mathrm{u}, v$ - particle's velocity and $c$ - the speed of light.

A comparison between the experimentally determined effective track radius and the physical one will allow us to conclude about the effectiveness of biological effects that can also contribute to the experimentally observed curvature of the dose effect curves. For example, in the case of $150 \mathrm{MeV}$ proton irradiation at $2 \mathrm{~Gy}$, the corresponding fluence amounts to about $22 \times 10^{8} 1 / \mathrm{cm}^{2}$ and $550 \mathrm{hits} / \mathrm{cell}$, which lead to the average distance between 

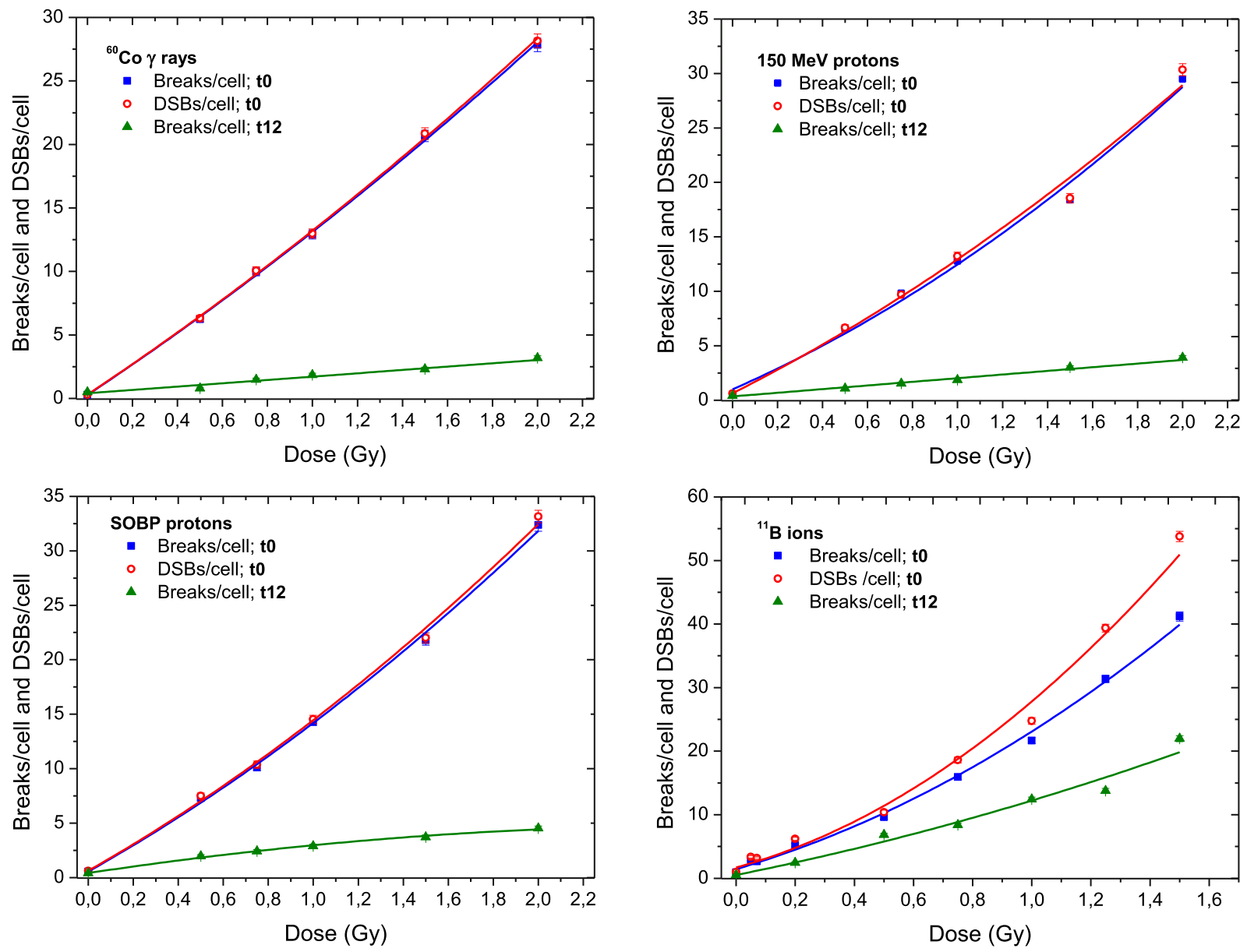

Fig. 2. Dose effect curves of breaks (chromatid and isochromatid), and DSBs induced by ${ }^{60} \mathrm{Co} \gamma \mathrm{rays}, 150 \mathrm{MeV}$ and $\mathrm{SOBP}$ protons and ${ }^{11} \mathrm{~B}$ ions measured immediately after exposure and 12 hours later. Additionally, breaks at $\mathrm{t}_{0}$ were recalculated into DSBs according to the scheme: one chromatid break corresponds to one DSB and one isochromatid break (excess figure) corresponds to two DSBs. Error bars are only of statistical origin.

hits of $200 \mathrm{~nm}$. It is to compare to the $R_{\text {phys }}=23 \mathrm{~nm}$. According to equation (2), the contribution from the overlapping tracks to the total dose is then small but not negligible. The resulting curvature ratio $\beta / \alpha$ takes the value of about $0.01 \mathrm{~Gy}^{-1}$.

\section{Results}

\subsection{Experimental results}

The chemically induced PCC technique was used to determine the yield of chromosome breakage and to measure the repair impact at two different times after the irradiation: immediately after the exposure (the time $t_{0}$ ) and after 12 hours (the time $\mathrm{t}_{12}$ ).

$\mathrm{G}_{2}$ cells analyzed at $t_{0}$ were at the same phase during irradiation, thus we could easily distinguish between the damage one of two sister chromatids (chromatid break, resulting from one DSB) and damage of both chromatids (excess figures), resulting from two DSBs).

$\mathrm{G}_{2}$ cells analyzed at $\mathrm{t}_{12}$ are a mixture of cells which at the moment of irradiation were in $\mathrm{G}_{1}$ or S-phase. Thus, observed breaks and excess figures might have been either the result of 1 DSB produced in $\mathrm{G}_{1}$ or 2 DSBs produced in the late $\mathrm{S}$ phase or a mixture of these events in earlymiddle S-phase.

In Figure 2, the dose-effect curves of all observed breaks at $t_{0}$ and $t_{12}$ are depicted. The corresponding fitted parameters $\alpha$ and $\beta$ are given in Table 1. Additionally, at $\mathrm{t}_{0}$, when the cells irradiated in $\mathrm{G}_{2}$ were immediately analyzed at the same phase, we can recalculate chromatid breaks into DSBs correlating one chromatid break with one DSB, excess figure (isochromatid break) with two DSBs (Fig. 2). For $t_{12}$ both chromatid breaks and excess figures were assumed to be the result of 1 DSB to avoid the overestimation of damage.

Only in the case of ${ }^{11} \mathrm{~B}$ ions, the curve representing DSBs/cell is significantly higher than the curve corresponding to the number of breaks/cell. It is due to the higher number of excess figures induced by ${ }^{11} \mathrm{~B}$ ions having the highest LET $(76 \mathrm{keV} / \mu \mathrm{m})$ among irradiation species being studied.

All $t_{0}$ dose effect curves possess a statistically significant quadratic contribution - the smallest one for ${ }^{60} \mathrm{Co}$ $\gamma$ rays and the largest for ${ }^{11} \mathrm{~B}$ ions. For the time $\mathrm{t}_{12}$, the 
Table 1. Parameters of the linear-quadratic fit $\left(Y=\alpha D+\beta D^{2}\right)$ of PCC breaks observed immediately after irradiation ( $\left.\mathrm{t}_{0}\right)$ and twelve hours after the exposure $\left(\mathrm{t}_{12}\right)$.

\begin{tabular}{lccccrr}
\hline & \multicolumn{5}{c}{$\mathrm{t}_{0}$} & \multicolumn{1}{c}{$\mathrm{t}_{12}$} \\
\cline { 2 - 6 } & $\alpha\left[G y^{-1}\right]$ & $\beta\left[G y^{-2}\right]$ & $\beta / \alpha\left[G y^{-1}\right]$ & $\alpha\left[G y^{-1}\right]$ & $\beta\left[G y^{-2}\right]$ & $\beta / \alpha\left[G y^{-1}\right]$ \\
\hline${ }^{60}$ Co $\gamma$ rays & $11.8 \pm 0.4$ & $1.04 \pm 0.29$ & $0.09 \pm 0.03$ & $0.99 \pm 0.21$ & $0.19 \pm 0.12$ & $0.19 \pm 0.13$ \\
$150 \mathrm{MeV}$ protons & $10.6 \pm 0.5$ & $1.6 \pm 0.3$ & $0.15 \pm 0.03$ & $1.27 \pm 0.21$ & $0.26 \pm 0.12$ & $0.21 \pm 0.10$ \\
SOBP protons & $11.7 \pm 0.5$ & $2.0 \pm 0.3$ & $0.17 \pm 0.03$ & $3.1 \pm 0.2$ & $-0.55 \pm 0.15$ & $-0.18 \pm 0.06$ \\
${ }^{11}$ B ions & $13.7 \pm 0.8$ & $8.0 \pm 0.7$ & $0.58 \pm 0.06$ & $9.4 \pm 0.5$ & $2.4 \pm 0.5$ & $0.25 \pm 0.05$ \\
\hline
\end{tabular}

Table 2. Values of the physical and effective track radii at $t_{0}$ and $\mathrm{t}_{12}$.

\begin{tabular}{lccc}
\hline \multirow{2}{*}{ Radiation; LET } & \multirow{2}{*}{$R_{\text {phys }}[\mathrm{nm}]$} & \multicolumn{2}{c}{$R_{\exp }[\mathrm{nm}]$} \\
\cline { 3 - 4 } & & $\mathrm{t}_{0}$ & $\mathrm{t}_{12}$ \\
\hline${ }^{60} \mathrm{Co} \gamma$ rays; $0.2 \mathrm{kev} / \mu \mathrm{m}$ & - & $43 \pm 6$ & $63 \pm 21$ \\
${ }^{150} \mathrm{MeV}$ protons; $0.57 \mathrm{kev} / \mu \mathrm{m}$ & 23 & $94 \pm 9$ & $110 \pm 30$ \\
$\mathrm{SOBP}$ protons; $1.4 \mathrm{kev} / \mu \mathrm{m}$ & 9.7 & $157 \pm 12$ & - \\
${ }^{11} \mathrm{~B}$ ions; $76 \mathrm{kev} / \mu \mathrm{m}$ & 11.0 & $2130 \pm 110$ & $1400 \pm 150$ \\
\hline
\end{tabular}

quadratic terms are much smaller and are of large statistical uncertainties, the largest $\beta$ parameter is observed for boron ions. It even becomes slightly negative in the case of SOBP protons, although biased by a large statistical uncertainty.

\subsection{Estimation of effective track radius}

The effective track radii (Eq. (2)) corresponding to the experimentally determined curvature of the dose effect curves are presented in Table 2 together with the physical expectations (Eq. (3)).

\section{Conclusions and discussion}

The main aim of the present work was to look for the contribution to the dose-effect curves resulting from the overlapping of ion tracks produced by charge particle irradiation in peripheral blood lymphocytes. This effect is usually dominated by the biological repair mechanisms as it is observed in chromosome aberrations (CA) studies $[14,23]$. To minimize the biological effects, we decided to apply the premature chromosome condensation (PCC) technique that allows to observe chromatid breaks immediately after exposure to ionizing radiation. The lymphocytes were irradiated by $150 \mathrm{MeV}$ and SOBP protons as well as boron ions and for comparison by ${ }^{60} \mathrm{Co} \gamma$ rays at doses up to $2 \mathrm{~Gy}$, allowing to determine corresponding dose-effect curves. Similar to the CA investigations, the dose-effect curves for chromatid breaks have a linear-quadratic shape, and the corresponding $\alpha$ and $\beta$ parameters could be determined at two different times after irradiation: immediately after exposure and 12 hours later. This procedure was chosen to estimate efficiency of the repair mechanisms which are strongly time dependent.

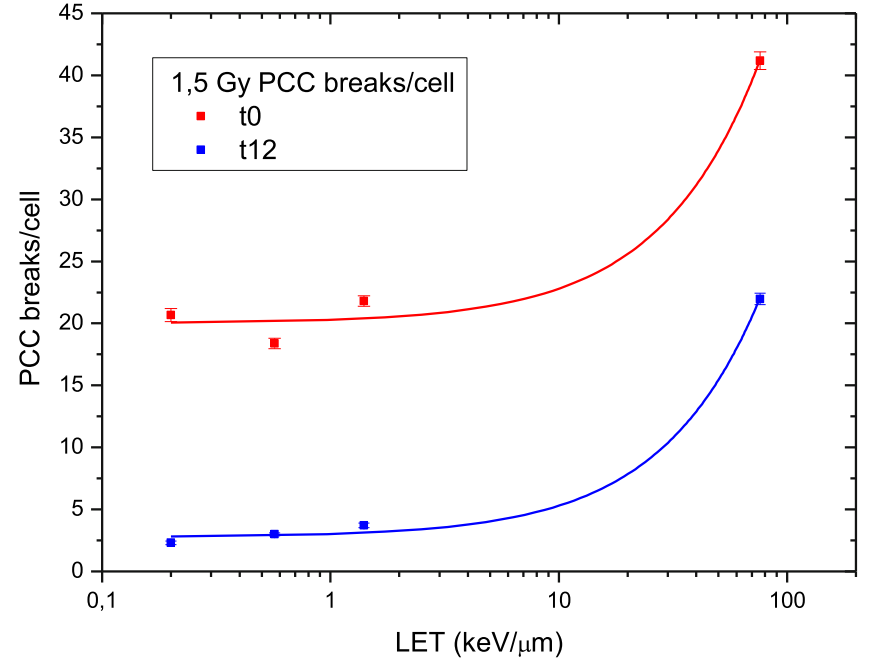

Fig. 3. Number of breaks per cell (described mainly by the $\alpha$ parameter) as a function of LET determined for $1.5 \mathrm{~Gy}$. The full lines represent linear fits.

It should be, however, noted that the DNA condensation is induced within 5 minutes after the Calyculin A application, and during this time interval necessary for DNA to condense, some fast rejoining/misrejoining of chromatid breaks occurs. A sign of this repair are exchange type aberrations few of which we observed at $t_{0}$ samples similar to those of the other authors [15,19]. From this point of view the "initial" yield of chromatid breaks in our case is actually "initially observed" rather than initially formed.

In Table 1, the parameters of the linear-quadratic model determined for both times are presented. Whereas the $\alpha$ parameter is proportional to the LET value of applied radiation (see Fig. 3) and describes its effectiveness for induction of chromatid breaks, the $\beta$ parameter results either from the ion track overlapping effect or from biological effects. The strength of the repair mechanisms can be easily estimated by means of reduction of the $\alpha$ parameter during 12 hours after irradiation. The largest decrease by a factor of about 10 is observed for high energy protons and gamma rays (low LET values) and the smallest one of 1.7 for boron ions (high LET value). This agrees with our expectation according to which the damage induced by boron ions is much more complex and difficult to repair. Their values are significantly reduced during 12 hours after irradiation. On the other hand, decrease of 
Table 3. Results of the ion track analysis performed for chromosome aberration in [14].

\begin{tabular}{lccccc}
\hline Irradiation; LET & $\begin{array}{c}R_{\min } \\
{[\mathrm{nm}]}\end{array}$ & $\begin{array}{c}R_{\max } \\
{[\mu \mathrm{m}]}\end{array}$ & $\begin{array}{c}R_{\text {phys }} \\
{[\mathrm{nm}]}\end{array}$ & $\begin{array}{c}\beta / \alpha \\
{\left[\mathrm{Gy}^{-1}\right]}\end{array}$ & $\begin{array}{c}R_{\text {exp }} \\
{[\mathrm{nm}]}\end{array}$ \\
\hline${ }^{60} \mathrm{Co} \gamma$ rays; $0.2 \mathrm{keV} / \mu \mathrm{m}$ & - & - & - & $1.6 \pm 0.5$ & $175 \pm 30$ \\
$150 \mathrm{MeV}$ protons; $0.57 \mathrm{keV} / \mu \mathrm{m}$ & 5.8 & 93 & 23.0 & $1.9 \pm 0.4$ & $318 \pm 25$ \\
$\mathrm{SOBP}$ protons; $1.4 \mathrm{keV} / \mu \mathrm{m}$ & 2.32 & 8.14 & 9.7 & $0.72 \pm 0.13$ & $287 \pm 28$ \\
${ }^{11} \mathrm{~B}$ ions; $76 \mathrm{keV} / \mu \mathrm{m}$ & 2.55 & 9.65 & 11.0 & - & - \\
\hline
\end{tabular}

the $\beta$ parameter is approximately the same for all radiation qualities and amount to $4.1 \pm 1.6$ (with exception of SOBP protons for which the $\mathrm{t}_{12}$ measurement at higher doses is probably biased by a systematic error and should be excluded from the analysis). Consequently, we observe that the curvature parameter $\beta / \alpha$ remains constant (or slightly increases) within the calculated statistical uncertainties for low LET values whereas for boron ions, the decrease by a factor $2.3 \pm 0.5$ is significant.

Within our effective track radius model, the experimentally determined $\beta / \alpha$ ratio is assessed to the track radii which can be then compared to the physically expected values (see Tab. 2). In absence of any biological effects, they should be independent of the cell cycle phase and irradiation time. The experimental values are, however, much larger than the theoretical ones and change visibly during 12 hours in the case of the boron exposure. This suggests that the repair mechanisms overestimate the track overlapping effect even at the time $t_{0}$, which is not surprising because of irradiation time of about 2 minutes and 5 minutes for the condensation of the DNA by application of Calyculin A. It roughly corresponds to the time constant of the short-term component of the repair mechanisms which is very effective within first 5-7 minutes [25-27].

Otherwise, it is also interesting to compare the present results obtained for chromatid breaks at $t_{12}$ with those obtained previously in the study of chromosome aberrations for the same ion beams (see Tab. 3). The experimental effective ion track radii for $\mathrm{CA}$ are larger by a factor 3-4 supposing larger repair mechanism contribution, which is in agreement with a long incubation time of irradiated lymphocytes of 48 hours and slightly increasing $\beta / \alpha$ ratios of chromatid response functions obtained for low LET values. An opposite tendency can be observed for the high LET irradiation of boron ions for which a strong decrease of the curvature parameter is found. Therefore, this effect might explain the parabolic shape of chromosome response functions observed for low LET radiations in contrast to a linear dependence for high LET values. This conclusion should be considered with great caution since in our CA experiments, we investigated lymphocytes in $\mathrm{G}_{0}$ phase which is of different radiosensitivity than the $\mathrm{G}_{2}$ phase. Furthermore, kinetics of repair mechanisms can differ in different cell cycle stages, as well. In $\mathrm{G}_{0}$ irradiated cells exchange aberrations dominate while irradiation closer to mitosis results mainly in breaks. Thus, in the PCC spreads at $t_{12}$, we do not observe typical exchange aberrations i.e. dicentrics except of few chromatid exchanges. Moreover, not all cells scored in the PCC assay would reach mitosis due to complicate cell cycle regulation [24].

Applying the PCC technique, we aimed to minimalize influence of biological repair mechanisms and make visible the physical effect of overlapping ion tracks. However, we have seen that even at our initial time $t_{0}$, the measurements are biased by the biological effects. The best method to overcome this difficulty seems to be application of Calyculin A several minutes before the irradiation, the starting point of which should be determined very precisely.

\section{Author contribution statement}

A. Kowalska and K. Czerski were mainly responsible for modelling and calculations while E. Nasonova, P. Kutsalo and E. Krasavin were mainly responsible for breaks scoring.

Open Access This is an open access article distributed under the terms of the Creative Commons Attribution License (http://creativecommons.org/licenses/by/4.0/), which permits unrestricted use, distribution, and reproduction in any medium, provided the original work is properly cited.

\section{References}

1. D. Schardt, T. Elsasser, D. Schulz-Ertner, Rev. Mod. Phys. 82, 383 (2010)

2. P.K. Agrawala, J.S. Adhikari, N.K. Chaudhury, J. Pharm. Bioallied Sci. 2, 197 (2010)

3. IAEA, Cytogenetic Dosimetry: Application in Preparedness for and Response to Radiation Emergencies (IAEA, Vienna, 2011)

4. D.E. Lea, Actions of Radiations on Living Cells (Cambridge University Press, Cambridge, 1955)

5. M. Rall, D. Kraft, M. Volcic, A. Cucu, E. Nasonova, G. Taucher-Scholz, H. Böning, L. Weismüller, C. Fournier, Front. Oncol. 4, 1 (2015)

6. G. Iliakis, H. Wang, A.R. Perrault, W. Boecker, B. Rosidi, F. Windhofer, W. Wu, J. Guan, G. Terzoudi, G. Pantelias, Cytogenet. Genome Res. 104, 14 (2004)

7. B.D. Loucas, M. Durante, S.M. Bailey, M.N. Cornforth, Radiat. Res. 179, 9 (2013)

8. E. Surdutovich, A.V. Solov'yov, Eur. Phys. J. D 72, 140 (2018)

9. G. Schiwietz, K. Czerski, M. Roth, F. Staunfenbiel, P.L. Grande, Nucl. Instrum. Methods Phys. Res. B 226, 683 (2004) 
10. A. Schipler, G. Iliakis, Nucleic Acids Res. 41, 7589 (2013)

11. T. Elsässer, M. Krämer, M. Scholz, Int. J. Radiat. Oncol. Biol. Phys. 71, 866 (2008)

12. R. Katz, B. Ackerson, M. Homayoonfar, S.C. Sharma, Radiat. Res. 47, 402 (1971)

13. R. Katz, Nucl. Track Detect. 2, 1 (1978)

14. A. Kowalska, K. Czerski, E. Nasonova, P. Kutsalo, E. Krasavin, Eur. Phys. J. D 71, 332 (2017)

15. M. Duranet et al., Radiat. Res. 149, 68 (1998)

16. G. Taucher-Scholz, J. Heilmann, M. Schneider, G. Kraft, Radiat. Environ. Biophys. 34, 101 (1995)

17. V. Valdiglesias, S. Giunta, M. Fenech, M. Neri, S. Bonassi, Mutat. Res. 753, 24 (2013)

18. E.J. Gotoh, J. Cell Physiol. 209, 297 (2006)

19. E.J. Gotoh, T. Kawata, M. Durante, Int. J. Radiat. Biol. 75, $1129(1999)$
20. A. Kowalska, E. Nasonova, K. Czerski, P. Kutsalo, W. Pereira, E. Krasavin, Radiat. Environ. Biophys. 58, 99 (2019)

21. A.A. Bezbakh, V.B. Zager, G. Kamiński, A.I. Krylov, Y.G. Teterev, G.N. Timoshenko, Phys. Part. Nucl. Lett. 10, 175 (2013)

22. A.Chatterjee, H.J. Schaefer, Radiat. Environ. Biophys. 13, 215 (1976)

23. M. Scholz, in Microdosimetric response of physical and biological systems to low- and high- LET radiations: theory and applications to dosimetry, edited by Y. Horovitz, 1st edn. (Elsevier, Amsterdam, 2006)

24. G.I. Terzoudi et al., Int. J. Radiat. Biol. 76, 607 (2000)

25. T. Kawata et al., Adv. Space Res. 27, 383 (2001)

26. T. Kawata et al., Int. J. Radiat. Biol. 76, 929 (2000)

27. A. Vral et al., Int. J. Radiat. Biol. 78, 249 (2002)

28. J.R.K. Savage, J. Med. Genet. 13, 103 (1976) 